Groups Geom. Dyn. 5 (2011), 231-250

DOI $10.4171 / \mathrm{GGD} / 125$
Groups, Geometry, and Dynamics

(C) European Mathematical Society

\title{
The moduli space of Keum-Naie surfaces
}

\author{
Ingrid Bauer and Fabrizio Catanese* \\ Alles Gute zum 60. Geburtstag, Fritz! $!^{\dagger}$
}

\begin{abstract}
Using a new description of the surfaces discovered by Keum and later investigated by Naie, and of their fundamental group, we prove the following main result.

Let $S$ be a smooth complex projective surface which is homotopically equivalent to a Keum-Naie surface. Then $S$ is a Keum-Naie surface. The connected component of the Gieseker moduli space corresponding to Keum-Naie surfaces is irreducible, normal, unirational of dimension 6.
\end{abstract}

Mathematics Subject Classification (2010). 14J29, 14J25, 14J10, 14D22, 14E20, 20F34, 32G05, 32Q30.

Keywords. Algebraic surfaces, moduli spaces, homotopy type, fundamental groups.

\section{Introduction}

In the nineties Y. H. Keum and D. Naie (cf. [Nai94], [Ke]) constructed a family of minimal surfaces of general type with $K_{S}^{2}=4$ and $p_{g}=0$ as double covers of an Enriques surface with eight nodes.

They calculated the fundamental group of the constructed surfaces, but they did not address the problem of determining the moduli space of their surfaces.

The motivation for the present paper comes from our joint work [BCGP09] together with F. Grunewald and R. Pignatelli. In that article, among other results, we constructed several series of new surfaces of general type with $p_{g}=0$ as minimal resolutions of quotients of a product of two curves (of respective genera $g_{1}, g_{2}$ at least two) by the action of a finite group $G$. This construction produced many interesting examples of new fundamental groups (of surfaces of general type with $p_{g}=0$ ) but in general yields proper subfamilies and not full irreducible components of the respective moduli spaces of surfaces of general type (see also [BC10], [BC11]).

\footnotetext{
*The present work took place in the realm of the DFG Forschergruppe 790 "Classification of algebraic surfaces and compact complex manifolds".

'Our wishes did not come through, and we regret very much the loss of an excellent mathematician, an exceptional person and a dear friend.
} 
Obviously, when two such families yield surfaces with non-isomorphic fundamental groups, then clearly the two families lie in distinct connected components of the moduli space. But what happens if the fundamental groups are isomorphic (and the value of $K_{S}^{2}$ is the same)?

In particular, two of the families we constructed in [BCGP09] corresponded to surfaces having the same fundamental group as the Keum-Naie surfaces. ${ }^{1}$

We reproduce below an excerpt of the classification table (of quotients as above by a non free action of $G$, but with canonical singularities) in [BCGP09].

\begin{tabular}{|c|c|c|c|c|c|c|c|}
\hline$K^{2}$ & $T_{1}$ & $T_{2}$ & $g_{1}$ & $g_{2}$ & $\mathrm{G}$ & $\operatorname{dim}$ & $\pi_{1}(S)$ \\
\hline \hline 4 & $2^{2}, 4^{2}$ & $2^{2}, 4^{2}$ & 3 & 3 & $\mathbb{Z} / 4 \mathbb{Z} \times \mathbb{Z} / 2 \mathbb{Z}$ & 2 & $\mathbb{Z}^{4} \hookrightarrow \pi_{1} \rightarrow(\mathbb{Z} / 2 \mathbb{Z})^{2}$ \\
\hline 4 & $2^{5}$ & $2^{5}$ & 3 & 3 & $(\mathbb{Z} / 2 \mathbb{Z})^{3}$ & 4 & $\mathbb{Z}^{4} \hookrightarrow \pi_{1} \rightarrow(\mathbb{Z} / 2 \mathbb{Z})^{2}$ \\
\hline
\end{tabular}

This excerpt shows the 2 families, of respective dimensions 2 and 4 , that we constructed as $\mathbb{Z} / 4 \mathbb{Z} \times \mathbb{Z} / 2 \mathbb{Z}$ - resp. $(\mathbb{Z} / 2 \mathbb{Z})^{3}$-coverings of $\mathbb{P}^{1} \times \mathbb{P}^{1}$ and branched on a divisor of type $(4,4)$ resp. $(5,5)$ which are the union of horizontal and vertical lines ( $T_{1}, T_{2}$ stand for the type of branching on each line).

Once we found out that their fundamental groups were isomorphic to the fundamental groups of the surfaces constructed by Keum and Naie, the most natural question was whether all these surfaces would belong to a unique irreducible component of the moduli space.

A straightforward computation showed that our family of dimension 4 was equal to the family constructed by Keum, and that both families were subfamilies of the family constructed by Naie. To be more precise, each surface of our family of $(\mathbb{Z} / 2 \mathbb{Z})^{3}$ coverings of $\mathbb{P}^{1} \times \mathbb{P}^{1}$ has four nodes. These nodes can be smoothened simultaneously, thus obtaining a 5 -dimensional family of $(\mathbb{Z} / 2 \mathbb{Z})^{3}$-Galois coverings of $\mathbb{P}^{1} \times \mathbb{P}^{1}$. The full 6-dimensional component is obtained then as the family of natural deformations (see [Cat08]) of the family of such Galois coverings.

A somewhat lengthy but essentially standard computation in local deformation theory showed that the 6-dimensional family of natural deformations of smooth $(\mathbb{Z} / 2 \mathbb{Z})^{3}$-Galois coverings of $\mathbb{P}^{1} \times \mathbb{P}^{1}$ is an irreducible component of the moduli space. We will not give the details of this calculation, since we get a stronger result by a different method.

The following theorem is the main result of this article:

Theorem 0.1. Let $S$ be a smooth complex projective surface which is homotopically equivalent to a Keum-Naie surface. Then $S$ is a Keum-Naie surface. The connected component of the Gieseker moduli space $\mathfrak{M}_{1,4}^{c a n}$ corresponding to Keum-Naie surfaces is irreducible, normal, unirational of dimension 6.

\footnotetext{
${ }^{1}$ Observe however that the correct description of the fundamental group is only to be found in [Nai94].
} 
Observe that for surfaces of general type we have two moduli spaces: one is the moduli space $\mathfrak{M}_{\chi, K^{2}}^{\min }$ for minimal models $S$ having $\chi\left(\mathcal{O}_{S}\right)=\chi, K_{S}^{2}=K^{2}$, the other is the moduli space $\mathfrak{M}_{\chi, K^{2}}^{\text {can }}$ for canonical models $X$ having $\chi\left(\mathcal{O}_{X}\right)=\chi$, $K_{X}^{2}=K^{2}$; the latter is called the Gieseker moduli space and is a quasi projective scheme by Gieseker's theorem ([Gie77]). Moreover, there is a natural morphism $\mathfrak{M}_{\chi, K^{2}}^{\min } \rightarrow \mathfrak{M}_{\chi, K^{2}}^{\mathrm{can}}$ which is a bijection. The local structure of $\mathfrak{M}_{\chi, K^{2}}^{\mathrm{can}}$ as complex analytic space is the quotient of the base of the Kuranishi family by the action of the finite group $\operatorname{Aut}(S)=\operatorname{Aut}(X)$.

Usually the structure as analytic space of $\mathfrak{M}_{\chi, K^{2}}^{\min }$ tends to be more singular than the one of $\mathfrak{M}_{\chi, K^{2}}^{\text {can }}$ (see e.g. [Cat89]).

In order to achieve our main result, we resort first of all to a slightly different construction of Keum-Naie surfaces.

We start with a $(\mathbb{Z} / 2 \mathbb{Z})^{2}$-action on the product of two elliptic curves $E_{1}^{\prime} \times E_{2}^{\prime}$.

This action has 16 fixed points and the quotient is an 8-nodal Enriques surface. Instead of constructing $S$ as the double cover of the Enriques surface, we consider an étale $(\mathbb{Z} / 2 \mathbb{Z})^{2}$-covering $\widehat{S}$ of $S$, whose existence is guaranteed from the structure of the fundamental group of $S$. $\widehat{S}$ is obtained as a double cover of $E_{1}^{\prime} \times E_{2}^{\prime}$ branched in a $(\mathbb{Z} / 2 \mathbb{Z})^{2}$-invariant divisor of type $(4,4)$, and $S$ is recovered as the quotient of $\hat{S}$ by the action of $(\mathbb{Z} / 2 \mathbb{Z})^{2}$ on it.

The structure of this $(\mathbb{Z} / 2 \mathbb{Z})^{2}$ action and the geometry of the covering $\hat{S}$ of $S$ is essentially encoded in the fundamental group $\pi_{1}(S)$, which is described as an affine group $\Gamma \in \mathbb{A}(2, \mathbb{C})$. In particular, it follows that the Albanese map of $\widehat{S}$ is the above double cover $\hat{\alpha}: \widehat{S} \rightarrow E_{1}^{\prime} \times E_{2}^{\prime}$.

If $S^{\prime}$ is now homotopically equivalent to a Keum-Naie surface $S$, then we have a corresponding étale $(\mathbb{Z} / 2 \mathbb{Z})^{2}$-covering $\hat{S}^{\prime}$ which is homotopically equivalent to $\hat{S}$. Since we know that the degree of the Albanese map of $\widehat{S}$ is equal to two (by construction), we can conclude the same for the Albanese map of $\hat{S}^{\prime}$ and this allows to deduce that also $\hat{S}^{\prime}$ is a double cover of a product of elliptic curves branched in a $(\mathbb{Z} / 2 \mathbb{Z})^{2}$-invariant divisor of type $(4,4)$.

Our paper is organized as follows: in Section 1 we study a certain $(\mathbb{Z} / 2 \mathbb{Z})^{2}$ action on a product of two elliptic curves $E_{1}^{\prime} \times E_{2}^{\prime}$ and explain our construction of Keum-Naie surfaces.

In Section 2 we use elementary representation theory to calculate the dimension of the space of $(\mathbb{Z} / 2 \mathbb{Z})^{2}$-invariant divisors of type $(4,4)$ on $E_{1}^{\prime} \times E_{2}^{\prime}$, and show that the Gieseker moduli space of Keum-Naie surfaces is a normal, irreducible, unirational variety of dimension six.

In Section 3 we conclude the proof of our main result 0.1

The brief Section 4 is devoted to the bicanonical image of Keum-Naie surfaces: we show that the map has degree 4 and that the image is always the same 4-nodal Del Pezzo surface of degree 4.

We stick to the traditional ('old fashioned'?) notation $\equiv$ to denote linear equivalence. 


\section{A $(\mathbb{Z} / 2 \mathbb{Z})^{2}$-action on a product of elliptic curves and Keum-Naie surfaces}

Let $(E, o)$ be any elliptic curve, with an action of the group

$$
G:=(\mathbb{Z} / 2 \mathbb{Z})^{2}=\left\{0, g_{1}, g_{2}, g_{3}:=g_{1}+g_{2}\right\}
$$

given by

$$
g_{1}(z):=z+\eta, \quad g_{2}(z)=-z,
$$

where $\eta \in E$ is a 2-torsion point of $E$.

Remark 1.1. The effective divisor $[o]+[\eta] \in \operatorname{Div}^{2}(E)$ is invariant under $G$, hence the invertible sheaf $\mathcal{O}_{E}([o]+[\eta])$ carries a natural $G$-linearization.

In particular, $G$ acts on the vector space $H^{0}\left(E, \mathcal{O}_{E}([o]+[\eta])\right)$ which then splits as a direct sum

$$
H^{0}\left(E, \mathcal{O}_{E}([o]+[\eta])\right)=\bigoplus_{\chi \in G^{*}} H^{0}\left(E, \mathcal{O}_{E}([o]+[\eta])\right)^{\chi}
$$

of the eigenspaces corresponding to the characters $\chi$ of $G$. We shall use the selfexplanatory notation $H^{0}\left(E, \mathcal{O}_{E}([o]+[\eta])\right)^{+-}$for the eigenspace corresponding to the character $\chi$ such that $\chi\left(g_{1}\right)=1, \chi\left(g_{2}\right)=-1$.

We have the following:

Lemma 1.1. In the above setting we have

$$
H^{0}\left(E, \mathcal{O}_{E}([o]+[\eta])\right)^{+-}=H^{0}\left(E, \mathcal{O}_{E}([o]+[\eta])\right)^{-+}=0,
$$

and we have a splitting as a sum of two 1-dimensional eigenspaces:

$$
H^{0}\left(E, \mathcal{O}_{E}([o]+[\eta])\right)=H^{0}\left(E, \mathcal{O}_{E}([o]+[\eta])\right)^{++} \oplus H^{0}\left(E, \mathcal{O}_{E}([o]+[\eta])\right)^{--} .
$$

Proof. Obviously, since the $G$ linearization is obtained by considering the vector space of rational functions with polar divisor at most $[o]+[\eta]$, the subspace $H^{0}\left(E, \mathcal{O}_{E}([o]+[\eta])\right)^{++}$has dimension at least 1 . On the other hand, there are exactly two $G$ invariant divisors in the linear system $|[o]+[\eta]|$.

If $[P]+[Q] \in|[o]+[\eta]|$ is $G$ invariant, then $g_{1}([P]+[Q])=[P+\eta]+[Q+\eta]=$ $[P]+[Q]$, hence $[P+\eta]=[Q]$. Since $[P]+[Q] \equiv[o]+[\eta], P, Q$ are 2-torsion points of $E$ (which automatically implies $g_{2}([P]+[Q])=[-P]+[-Q]=[P]+[Q]$ ), and we have shown that there are exactly two $G$-invariant divisors.

Therefore $H^{0}\left(E, \mathcal{O}_{E}([o]+[\eta])\right)$ splits as the direct sum of two 1-dimensional eigenspaces, one of which is $H^{0}\left(E, \mathcal{O}_{E}([o]+[\eta])\right)^{++}$.

It suffices now to show that

$$
H^{0}\left(E, \mathcal{O}_{E}([o]+[\eta])\right)^{+-}=H^{0}\left(E, \mathcal{O}_{E}([o]+[\eta])\right)^{-+}=0 .
$$


In fact, if this were not the case, all the divisors in the linear system $|[o]+[\eta]|$ would be invariant by either $g_{1}$ or by $g_{2}$.

The first possibility was already excluded above, while the second one means that, for each point $P,[P]+[\eta-P] \in|[o]+[\eta]|$ satisfies $g_{2}([P]+[\eta-P])=$ $[-P]+[P-\eta]=[P]+[\eta-P]$, which implies $[P]=[-P]$, a contradiction.

Consider now two complex elliptic curves $E_{1}^{\prime}, E_{2}^{\prime}$, which can be written as quotients $E_{i}^{\prime}:=\mathbb{C} / \Lambda_{i}^{\prime}, i=1,2$, with $\Lambda_{i}^{\prime}:=\mathbb{Z} e_{i} \oplus \mathbb{Z} e_{i}^{\prime}$.

We consider the affine transformations $\gamma_{1}, \gamma_{2} \in \mathbb{A}(2, \mathbb{C})$, defined as follows:

$$
\gamma_{1}\left(\begin{array}{c}
z_{1} \\
z_{2}
\end{array}\right):=\left(\begin{array}{c}
z_{1}+\frac{e_{1}}{2} \\
-z_{2}
\end{array}\right), \quad \gamma_{2}\left(\begin{array}{c}
z_{1} \\
z_{2}
\end{array}\right):=\left(\begin{array}{c}
-z_{1} \\
z_{2}+\frac{e_{2}}{2}
\end{array}\right),
$$

and let $\Gamma \leq \mathbb{A}(2, \mathbb{C})$ be the affine group generated by $\gamma_{1}, \gamma_{2}$ and by the translations $e_{1}, e_{1}^{\prime}, e_{2}, e_{2}^{\prime}$.

Remark 1.2. i) $\Gamma$ contains the lattice $\Lambda_{1}^{\prime} \oplus \Lambda_{2}^{\prime}$, hence $\Gamma$ acts on $E_{1}^{\prime} \times E_{2}^{\prime}$ inducing a faithful action of $G:=(\mathbb{Z} / 2 \mathbb{Z})^{2}$ on $E_{1}^{\prime} \times E_{2}^{\prime}$.

ii) While $\gamma_{1}, \gamma_{2}$ have no fixed points on $E_{1}^{\prime} \times E_{2}^{\prime}$, the involution $\gamma_{1} \gamma_{2}$ has 16 fixed points on $E_{1}^{\prime} \times E_{2}^{\prime}$. It is easy to see that the quotient $Y:=\left(E_{1}^{\prime} \times E_{2}^{\prime}\right) / G$ is an Enriques surface having eight nodes, with canonical double cover the Kummer surface $\left(E_{1}^{\prime} \times E_{2}^{\prime}\right) /\left\langle\gamma_{1} \gamma_{2}\right\rangle$.

We will in the sequel lift the $G$-action on $E_{1}^{\prime} \times E_{2}^{\prime}$ to an appropriate ramified double cover $\widehat{S}$ and in such a way that $G$ acts freely on $\widehat{S}$.

Consider the geometric line bundle $\mathbb{L}$ on $E_{1}^{\prime} \times E_{2}^{\prime}$, whose invertible sheaf of sections is given by

$$
\mathcal{O}_{E_{1}^{\prime} \times E_{2}^{\prime}}(\mathbb{L}):=p_{1}^{*} \mathcal{O}_{E_{1}^{\prime}}\left(\left[o_{1}\right]+\left[\frac{e_{1}}{2}\right]\right) \otimes p_{2}^{*} \mathcal{O}_{E_{2}^{\prime}}\left(\left[o_{2}\right]+\left[\frac{e_{2}}{2}\right]\right),
$$

where $p_{i}: E_{1}^{\prime} \times E_{2}^{\prime} \rightarrow E_{i}^{\prime}$ is the projection onto the $i$-th factor.

Remark 1.3. By Remark 1.1, the divisor $\left[o_{i}\right]+\left[\frac{e_{i}}{2}\right] \in \operatorname{Div}^{2}\left(E_{i}^{\prime}\right)$ is invariant under $G$. Whence, we get a natural $G$-action on $\mathbb{L}$. But this is not the $G$-action on $\mathbb{L}$ that we shall consider.

In fact, any two $G$-actions on $\mathbb{L}$ differ by a character $\chi: G \rightarrow \mathbb{C}^{*}$. We shall twist the above natural action of $\mathbb{L}$ by the character such that $\chi\left(\gamma_{1}\right)=1, \chi\left(\gamma_{2}\right)=-1$. We shall call this twisted $G$-action the canonical one.

Definition 1.2. Consider the canonical $G$-action on $\mathbb{L}$ and on all its tensor powers, and let

$$
f \in H^{0}\left(E_{1}^{\prime} \times E_{2}^{\prime}, p_{1}^{*} \mathcal{O}_{E_{1}^{\prime}}\left(2\left[o_{1}\right]+2\left[\frac{e_{1}}{2}\right]\right) \otimes p_{2}^{*} \mathcal{O}_{E_{2}^{\prime}}\left(2\left[o_{2}\right]+2\left[\frac{e_{2}}{2}\right]\right)\right)^{G}
$$

be a $G$-invariant section of $\mathbb{L}^{\otimes 2}$. 
Denoting by $w$ a fibre coordinate of $\mathbb{L}$, let $\widehat{X}$ be the double cover of $E_{1}^{\prime} \times E_{2}^{\prime}$ branched in $\{f=0\}$, i.e., set

$$
\widehat{X}=\left\{w^{2}=f\left(z_{1}, z_{2}\right)\right\} \subset \mathbb{L} .
$$

Then $\hat{X}$ is a $G$-invariant hypersurface in $\mathbb{L}$, and we define the canonical model of a Keum-Naie surface to be the quotient of $\hat{X}$ by the $G$-action.

More precisely, we define $S$ to be a Keum-Naie surface, if

- $G$ acts freely on $\hat{X}$, and

- $\{f=0\}$ has only non-essential singularities, i.e., $\widehat{X}$ has canonical singularities (at most rational double points);

- $S$ is the minimal resolution of singularities of $X:=\hat{X} / G$.

Remark 1.4. One might also call the above surfaces 'primary Keum-Naie surfaces'. In fact a similar construction, applied to the case where the action of $G$ has fixed points at some nodal singularities of some special $\hat{X}$, produces other surfaces, which could appropriately be named 'secondary Keum-Naie surfaces'.

Lemma 1.3. If

$$
f \in H^{0}\left(E_{1}^{\prime} \times E_{2}^{\prime}, p_{1}^{*} \mathcal{O}_{E_{1}^{\prime}}\left(2\left[o_{1}\right]+2\left[\frac{e_{1}}{2}\right]\right) \otimes p_{2}^{*} \mathcal{O}_{E_{2}^{\prime}}\left(2\left[o_{2}\right]+2\left[\frac{e_{2}}{2}\right]\right)\right)^{G}
$$

is such that $\left\{\left(z_{1}, z_{2}\right) \in E_{1}^{\prime} \times E_{2}^{\prime} \mid f\left(z_{1}, z_{2}\right)=0\right\} \cap \operatorname{Fix}\left(\gamma_{1} \gamma_{2}\right)=\emptyset$, then $G$ acts freely on $\hat{X}$.

Proof. Recall that $\gamma_{1}, \gamma_{2}$ do not have fixed points on $E_{1}^{\prime} \times E_{2}^{\prime}$, whence they have no fixed points on $\hat{X}$. Since, by $1.3,\left(\gamma_{1} \gamma_{2}\right)(w)=-w$, it follows that $G$ acts freely on $\widehat{X}$ if and only if $\{f=0\}$ does not intersect the fixed points of $\gamma_{1} \gamma_{2}$ on $E_{1}^{\prime} \times E_{2}^{\prime}$.

Proposition 1.4. Let $S$ be a Keum-Naie surface. Then $S$ is a minimal surface of general type with

i) $K_{S}^{2}=4$,

ii) $p_{g}(S)=q(S)=0$,

iii) $\pi_{1}(S)=\Gamma$.

Proof. i) Let $\pi: \hat{X} \rightarrow E_{1}^{\prime} \times E_{2}^{\prime}$ be the above double cover branched on $\{f=0\}$. Then $K_{\hat{X}} \equiv \pi^{*}\left(K_{E_{1}^{\prime} \times E_{2}^{\prime}}+p_{1}^{*}\left(\left[o_{1}\right]+\left[\frac{e_{1}}{2}\right]\right)+p_{2}^{*}\left(\left[o_{2}\right]+\left[\frac{e_{2}}{2}\right]\right)\right)$, whence $K_{\hat{X}}^{2}=$ $2 \cdot\left(p_{1}^{*}\left(\left[o_{1}\right]+\left[\frac{e_{1}}{2}\right]\right)+p_{2}^{*}\left(\left[o_{2}\right]+\left[\frac{e_{2}}{2}\right]\right)\right)^{2}=2 \cdot 8=16$. Therefore $K_{S}^{2}=K_{X}^{2}=\frac{K_{\hat{X}}^{2}}{|G|}=4$.

ii) Let $\sigma: \hat{S} \rightarrow \widehat{X}$ be the minimal resolution of singularities of $\hat{X}$. Then $S=$ $\widehat{S} / G$, and

$$
H^{0}\left(S, \Omega_{S}^{1}\right)=H^{0}\left(\widehat{S}, \Omega_{\widehat{S}}^{1}\right)^{G}
$$


Since $\pi \circ \sigma: \widehat{S} \rightarrow E_{1}^{\prime} \times E_{2}^{\prime}$ has degree 2, it is the Albanese map of $\widehat{S}$, and we have that $H^{0}\left(\widehat{S}, \Omega_{\widehat{S}}^{1}\right)=H^{0}\left(E_{1}^{\prime} \times E_{2}^{\prime}, \Omega_{E_{1}^{\prime} \times E_{2}^{\prime}}^{1}\right) \cong \mathbb{C} d z_{1} \oplus \mathbb{C} d z_{2}$. Hence

$$
H^{0}\left(S, \Omega_{S}^{1}\right)=H^{0}\left(\widehat{S}, \Omega_{\widehat{S}}^{1}\right)^{G}=0,
$$

i.e., $q(S)=0$.

Observe that since $G$ acts freely

$$
H^{0}\left(\hat{X}, \mathcal{O}\left(K_{\hat{X}}\right)\right)^{G}=H^{0}\left(X, \mathcal{O}\left(K_{X}\right)\right)=H^{0}\left(S, \Omega_{S}^{2}\right) .
$$

Consider now the decomposition of

$$
V:=H^{0}\left(\widehat{X}, \mathcal{O}\left(K_{\widehat{X}}\right)\right)=H^{0}\left(\widehat{X}, \mathcal{O}\left(K_{\widehat{X}}\right)\right)^{+} \oplus H^{0}\left(\widehat{X}, \mathcal{O}\left(K_{\widehat{X}}\right)\right)^{-}
$$

in the invariant and anti-invariant part for the action of the involution $\sigma$ of the double cover $\pi: \hat{X} \rightarrow E_{1}^{\prime} \times E_{2}^{\prime}\left(\sigma\left(z_{1}, z_{2}, w\right)=\left(z_{1}, z_{2},-w\right)\right)$.

Note that

a) $H^{0}\left(\hat{X}, \mathcal{O}\left(K_{\hat{X}}\right)\right)^{+}=H^{0}\left(E_{1}^{\prime} \times E_{2}^{\prime}, \Omega_{E_{1}^{\prime} \times E_{2}^{\prime}}^{2}\right)=\mathbb{C}\left(d z_{1} \wedge d z_{2}\right)$,

b) $H^{0}\left(\hat{X}, \mathcal{O}\left(K_{\hat{X}}\right)\right)^{-} \cong H^{0}\left(E_{1}^{\prime} \times E_{2}^{\prime}, \Omega_{E_{1}^{\prime} \times E_{2}^{\prime}}^{2}(\mathbb{L})\right)$.

In the uniformizing coordinates the first summand a) is generated by $d z_{1} \wedge d z_{2}$, which is an eigenvector for the $G$-action, with character $\chi$ such that $\chi\left(\gamma_{1}\right)=\chi\left(\gamma_{2}\right)=$ -1 . We shall call this eigenspace $V^{--}$.

Each vector $y$ in the addendum b) can be written as

$$
y=\frac{\varphi_{1}\left(z_{1}\right) \varphi_{2}\left(z_{2}\right)}{w} d z_{1} \wedge d z_{2},
$$

where $\varphi_{i} \in H^{0}\left(E_{i}^{\prime}, \mathcal{O}_{E_{i}^{\prime}}\left(\left[o_{i}\right]+\left[\frac{e_{i}}{2}\right]\right)\right)$.

Recall that (cf. Lemma 1.1) $H^{0}\left(E_{i}^{\prime}, \mathcal{O}_{E_{i}^{\prime}}\left(\left[o_{i}\right]+\left[\frac{e_{i}}{2}\right]\right)\right)=: H_{i}$ splits as $H_{i}^{++} \oplus$ $H_{i}^{--}$(observe that exchanging the roles of $g_{1}$ and $g_{2}$ in Lemma 1.1 makes fortunately no difference).

Using that $\gamma_{1}(w)=w, \gamma_{2}(w)=-w$ and that $d z_{1} \wedge d z_{2} \in V^{--}$, we get:

$$
\begin{array}{r}
\frac{\varphi_{1}\left(z_{1}\right) \varphi_{2}\left(z_{2}\right)}{w} d z_{1} \wedge d z_{2} \in V^{+-} \Longleftrightarrow \varphi_{1} \in H_{1}^{++} \wedge \varphi_{2} \in H_{2}^{--} \text {or } \\
\varphi_{1} \in H_{1}^{--} \wedge \varphi_{2} \in H_{2}^{++} ; \\
\frac{\varphi_{1}\left(z_{1}\right) \varphi_{2}\left(z_{2}\right)}{w} d z_{1} \wedge d z_{2} \in V^{-+} \Longleftrightarrow \varphi_{1} \in H_{1}^{++} \wedge \varphi_{2} \in H_{2}^{++} \text {or } \\
\varphi_{1} \in H_{1}^{--} \wedge \varphi_{2} \in H_{2}^{--} .
\end{array}
$$

The above calculations show that both eigenspaces $V^{-+}, V^{+-}$are 2-dimensional. Since the summand $b$ ) has dimension 4 , we obtain then: 
i) $H^{0}\left(\widehat{X}, \mathcal{O}\left(K_{\hat{X}}\right)\right)^{--}=\mathbb{C}\left(d z_{1} \wedge d z_{2}\right)$,

ii) $H^{0}\left(\hat{X}, \mathcal{O}\left(K_{\hat{X}}\right)\right)^{+-}=\left\{\frac{\varphi_{1}\left(z_{1}\right) \varphi_{2}\left(z_{2}\right)}{w} d z_{1} \wedge d z_{2} \mid\left(\varphi_{1} \in H_{1}^{++}\right.\right.$and $\left.\varphi_{2} \in H_{2}^{--}\right)$or $\left(\varphi_{1} \in H_{1}^{--}\right.$and $\left.\left.\varphi_{2} \in H_{2}^{++}\right)\right\}$has dimension 2;

iii) $H^{0}\left(\hat{X}, \mathcal{O}\left(K_{\hat{X}}\right)\right)^{-+}=\left\{\frac{\varphi_{1}\left(z_{1}\right) \varphi_{2}\left(z_{2}\right)}{w} d z_{1} \wedge d z_{2} \mid\left(\varphi_{1} \in H_{1}^{++}\right.\right.$and $\left.\varphi_{2} \in H_{2}^{++}\right)$ or $\left(\varphi_{1} \in H_{1}^{--}\right.$and $\left.\left.\varphi_{2} \in H_{2}^{--}\right)\right\}$has dimension 2 ;

iv) $H^{0}\left(\hat{X}, \mathcal{O}\left(K_{\hat{X}}\right)\right)^{++}=0$.

In particular, we get $p_{g}(S)=\operatorname{dim} H^{0}\left(\hat{X}, \mathcal{O}\left(K_{\hat{X}}\right)\right)^{++}=0$.

iii) it suffices to show that the fundamental group of $\widehat{S}$ maps isomorphically to the fundamental group of $E_{1}^{\prime} \times E_{2}^{\prime}$. By the theorem of Brieskorn-Tyurina ([Brie68], [Brie71], [Tju70]) we can reduce to the case where $\hat{X}$ is smooth, since the parameter space is connected, and there is a non empty open set of smooth branch curves $D$.

When $D$ is smooth, we conclude by the Lefschetz type theorem of Mandelbaum and Moishezon ([M-M80], p. 218), since $D$ is ample.

\section{The moduli space of Keum-Naie surfaces}

The aim of this section is to prove the following result.

Theorem 2.1. The connected component of the Gieseker moduli space corresponding to Keum-Naie surfaces is normal, irreducible, unirational of dimension equal to 6. Moreover, the base of the Kuranishifamily of the canonical model X of a Keum-Naie surface is smooth.

In order to describe the moduli space of Keum-Naie surfaces we shall preliminarily describe the vector space

$$
H^{0}\left(E_{1}^{\prime} \times E_{2}^{\prime}, p_{1}^{*} \mathcal{O}_{E_{1}^{\prime}}\left(2\left[o_{1}\right]+2\left[\frac{e_{1}}{2}\right]\right) \otimes p_{2}^{*} \mathcal{O}_{E_{2}^{\prime}}\left(2\left[o_{2}\right]+2\left[\frac{e_{2}}{2}\right]\right)\right)^{G} .
$$

We consider $E_{1}^{\prime}$ (resp. $E_{2}^{\prime}$ ) as a bidouble cover of $\mathbb{P}^{1}$ ramified in 4 points $\{0,1, \infty, P\}$ (resp. $\{0,1, \infty, Q\})$, where $G=(\mathbb{Z} / 2 \mathbb{Z})^{2}=\left\{0, g_{1}, g_{2}, g_{3}:=g_{1}+g_{2}\right\}$ acts as follows:

$$
\begin{array}{ll}
g_{1}(z)=z+\frac{e_{1}}{2}, & g_{2}(z)=-z \quad \text { on } E_{1}^{\prime}, \\
g_{1}(z)=-z, & g_{2}(z)=z+\frac{e_{2}}{2} \quad \text { on } E_{2}^{\prime} .
\end{array}
$$

We denote the respective bidouble covering maps from $E_{i}^{\prime}$ to $\mathbb{P}^{1}$ by $\pi_{i}$. Observe moreover that the quotient of $E_{1}^{\prime}$ by the action of $g_{1}$ is an elliptic curve $E_{1}$, while the quotient of $E_{1}^{\prime}$ by the action of $g_{2}$ (resp. $g_{3}$ ) is isomorphic to $\mathbb{P}^{1}$.

Remark 2.1. It is immediate from the above remark that the character eigensheaves of the direct image sheaf $\pi_{1 *} \mathcal{O}_{E_{1}^{\prime}}$ for the bidouble cover $\pi_{1}: E_{1}^{\prime} \rightarrow \mathbb{P}^{1}$ are

$$
\mathscr{L}_{1}^{-+}=\mathcal{O}_{\mathbb{P}^{1}}(1), \quad \mathscr{L}_{1}^{+-}=\mathcal{O}_{\mathbb{P}^{1}}(2), \quad \mathscr{L}_{1}^{--}=\mathcal{O}_{\mathbb{P}^{1}}(1)
$$


In fact, for instance, the direct image on $\mathbb{P}^{1}$ of the sheaf of functions on $E_{1}^{\prime} / g_{1}$ must be $\cong \mathcal{O}_{\mathbb{P}^{1}} \oplus \mathcal{O}_{\mathbb{P}^{1}}(-2)$ and it equals $\mathcal{O}_{\mathbb{P}^{1}} \oplus\left(\mathscr{L}_{1}^{+-}\right)^{-1}$.

Similarly for $\pi_{2}: E_{2}^{\prime} \rightarrow \mathbb{P}^{1}$ we have the character sheaves

$$
\mathscr{L}_{2}^{-+}=\mathcal{O}_{\mathbb{P}^{1}}(2), \quad \mathscr{L}_{2}^{+-}=\mathcal{O}_{\mathbb{P}^{1}}(1), \quad \mathscr{L}_{2}^{--}=\mathcal{O}_{\mathbb{P}^{1}}(1) .
$$

Since $\mathcal{O}_{E_{i}^{\prime}}\left(2\left[o_{i}\right]+2\left[\frac{e_{i}}{2}\right]\right)=\pi_{i}^{*}\left(\mathcal{O}_{\mathbb{P}^{1}}(1)\right)$ we get

$$
H^{0}\left(E_{i}^{\prime}, \mathcal{O}_{E_{i}^{\prime}}\left(2\left[o_{i}\right]+2\left[\frac{e_{i}}{2}\right]\right)\right)=H^{0}\left(\mathbb{P}^{1}, \mathcal{O}_{\mathbb{P}^{1}}(1) \otimes\left(\pi_{i}\right)_{*} \mathcal{O}_{E_{i}^{\prime}}\right),
$$

and therefore
i) $V_{1}^{++}:=H^{0}\left(E_{1}^{\prime}, \mathcal{O}_{E_{1}^{\prime}}\left(2\left[o_{1}\right]+2\left[\frac{e_{1}}{2}\right]\right)\right)^{++}=H^{0}\left(\mathbb{P}^{1}, \mathcal{O}_{\mathbb{P}^{1}}(1)\right) \cong \mathbb{C}^{2}$;
ii) $V_{1}^{+-}:=H^{0}\left(\mathcal{O}_{E_{1}^{\prime}}\left(2\left[o_{1}\right]+2\left[\frac{e_{1}}{2}\right]\right)\right)^{+-}=H^{0}\left(\mathcal{O}_{\mathbb{P}^{1}}(1) \otimes\left(\mathscr{L}_{1}^{+-}\right)^{-1}\right)=0$;
iii) $V_{1}^{-+}:=H^{0}\left(\mathcal{O}_{E_{1}^{\prime}}\left(2\left[o_{1}\right]+2\left[\frac{e_{1}}{2}\right]\right)\right)^{-+}=H^{0}\left(\mathcal{O}_{\mathbb{P}^{1}}(1) \otimes\left(\mathscr{L}_{1}^{-+}\right)^{-1}\right) \cong \mathbb{C}$;
iv) $V_{1}^{--}:=H^{0}\left(\mathcal{O}_{E_{1}^{\prime}}\left(2\left[o_{1}\right]+2\left[\frac{e_{1}}{2}\right]\right)\right)^{--}=H^{0}\left(\mathcal{O}_{\mathbb{P}^{1}}(1) \otimes\left(\mathscr{L}_{1}^{--}\right)^{-1}\right) \cong \mathbb{C}$;
v) $V_{2}^{++}:=H^{0}\left(E_{2}^{\prime}, \mathcal{O}_{E_{2}^{\prime}}\left(2\left[o_{2}\right]+2\left[\frac{e_{2}}{2}\right]\right)\right)^{++}=H^{0}\left(\mathbb{P}^{1}, \mathcal{O}_{\mathbb{P}^{1}}(1)\right) \cong \mathbb{C}^{2}$;
vi) $V_{2}^{+-}:=H^{0}\left(\mathcal{O}_{E_{2}^{\prime}}\left(2\left[o_{2}\right]+2\left[\frac{e_{2}}{2}\right]\right)\right)^{+-}=H^{0}\left(\mathcal{O}_{\mathbb{P}^{1}}(1) \otimes\left(\mathscr{L}_{2}^{+-}\right)^{-1}\right) \cong \mathbb{C}$;
vii) $V_{2}^{-+}:=H^{0}\left(\mathcal{O}_{E_{2}^{\prime}}\left(2\left[o_{2}\right]+2\left[\frac{e_{2}}{2}\right]\right)\right)^{-+}=H^{0}\left(\mathcal{O}_{\mathbb{P}^{1}}(1) \otimes\left(\mathscr{L}_{2}^{-+}\right)^{-1}\right)=0$;
viii) $V_{2}^{--}:=H^{0}\left(\mathcal{O}_{E_{2}^{\prime}}\left(2\left[o_{2}\right]+2\left[\frac{e_{2}}{2}\right]\right)\right)^{--}=H^{0}\left(\mathcal{O}_{\mathbb{P}^{1}}(1) \otimes\left(\mathscr{L}_{2}^{--}\right)^{-1}\right) \cong \mathbb{C}$.

As a consequence of the above remark, we get

\section{Lemma 2.2 .}

1) $H^{0}\left(E_{1}^{\prime} \times E_{2}^{\prime}, p_{1}^{*} \mathcal{O}_{E_{1}^{\prime}}\left(2\left[o_{1}\right]+2\left[\frac{e_{1}}{2}\right]\right) \otimes p_{2}^{*} \mathcal{O}_{E_{2}^{\prime}}\left(2\left[o_{2}\right]+2\left[\frac{e_{2}}{2}\right]\right)\right)^{++}$

$$
=\left(V_{1}^{++} \otimes V_{2}^{++}\right) \oplus\left(V_{1}^{--} \otimes V_{2}^{--}\right) \cong \mathbb{C}^{5} \text {; }
$$

2) $H^{0}\left(E_{1}^{\prime} \times E_{2}^{\prime}, p_{1}^{*} \mathcal{O}_{E_{1}^{\prime}}\left(2\left[o_{1}\right]+2\left[\frac{e_{1}}{2}\right]\right) \otimes p_{2}^{*} \mathcal{O}_{E_{2}^{\prime}}\left(2\left[o_{2}\right]+2\left[\frac{e_{2}}{2}\right]\right)\right)^{--}$

$$
=\left(V_{1}^{++} \otimes V_{2}^{--}\right) \oplus\left(V_{1}^{--} \otimes V_{2}^{++}\right) \oplus\left(V_{1}^{-+} \otimes V_{2}^{+-}\right) \cong \mathbb{C}^{5} ;
$$

Proof. This follows immediately from the above remark since

1) $H^{0}\left(E_{1}^{\prime} \times E_{2}^{\prime}, p_{1}^{*} \mathcal{O}_{E_{1}^{\prime}}\left(2\left[o_{1}\right]+2\left[\frac{e_{1}}{2}\right]\right) \otimes p_{2}^{*} \mathcal{O}_{E_{2}^{\prime}}\left(2\left[o_{2}\right]+2\left[\frac{e_{2}}{2}\right]\right)\right)^{G}$

$$
\begin{aligned}
& =\bigoplus_{\chi \in G^{*}}\left(H^{0}\left(\mathcal{O}_{E_{1}^{\prime}}\left(2\left[o_{1}\right]+2\left[\frac{e_{1}}{2}\right]\right)\right)^{\chi} \otimes H^{0}\left(E_{2}^{\prime}, \mathcal{O}_{E_{2}^{\prime}}\left(2\left[o_{2}\right]+2\left[\frac{e_{2}}{2}\right]\right)\right)^{\chi^{-1}}\right) \\
& =\left(V_{1}^{++} \otimes V_{2}^{++}\right) \oplus\left(V_{1}^{--} \otimes V_{2}^{--}\right) \cong \mathbb{C}^{4} \oplus \mathbb{C}
\end{aligned}
$$


and

$$
\text { 2) } \begin{aligned}
H^{0} & \left(E_{1}^{\prime} \times E_{2}^{\prime}, p_{1}^{*} \mathcal{O}_{E_{1}^{\prime}}\left(2\left[o_{1}\right]+2\left[\frac{e_{1}}{2}\right]\right) \otimes p_{2}^{*} \mathcal{O}_{E_{2}^{\prime}}\left(2\left[o_{2}\right]+2\left[\frac{e_{2}}{2}\right]\right)\right)^{--} \\
& =\bigoplus_{\chi \in G^{*}}\left(H^{0}\left(\mathcal{O}_{E_{1}^{\prime}}\left(2\left[o_{1}\right]+2\left[\frac{e_{1}}{2}\right]\right)\right)^{\chi} \otimes H^{0}\left(E_{2}^{\prime}, \mathcal{O}_{E_{2}^{\prime}}\left(2\left[o_{2}\right]+2\left[\frac{e_{2}}{2}\right]\right)\right)^{\chi^{-1} \chi^{\prime}}\right) \\
& =\left(V_{1}^{++} \otimes V_{2}^{--}\right) \oplus\left(V_{1}^{--} \otimes V_{2}^{++}\right) \oplus\left(V_{1}^{-+} \otimes V_{2}^{+-}\right) \cong \mathbb{C}^{2} \oplus \mathbb{C}^{2} \oplus \mathbb{C}
\end{aligned}
$$

where $\chi^{\prime}\left(g_{1}\right)=-1, \chi^{\prime}\left(g_{2}\right)=-1$.

Now we can conclude the proof of Theorem 2.1.

Proof of Theorem 2.1. Note that $V_{i}^{++}$is without base points, whence also $V_{1}^{++} \otimes$ $V_{2}^{++}$has no base points. Therefore a generic

$$
f \in H^{0}\left(E_{1}^{\prime} \times E_{2}^{\prime}, p_{1}^{*} \mathcal{O}_{E_{1}^{\prime}}\left(2\left[o_{1}\right]+2\left[\frac{e_{1}}{2}\right]\right) \otimes p_{2}^{*} \mathcal{O}_{E_{2}^{\prime}}\left(2\left[o_{2}\right]+2\left[\frac{e_{2}}{2}\right]\right)\right)^{G}
$$

has smooth and irreducible zero divisor $D$ (observe that $D$ is ample).

We obtain a 6-dimensional rational family parametrizing all the Keum-Naie surfaces simply by varying the two points $P, Q$ in $\mathbb{P}^{1} \backslash\{0,1, \infty\}$, and varying $f$ in an open set of the bundle of 4-dimensional projective spaces associated to the rank five vector bundle with fibre

$$
\left(V_{1}^{++} \otimes V_{2}^{++}\right) \oplus\left(V_{1}^{--} \otimes V_{2}^{--}\right) .
$$

We obtain an irreducible unirational algebraic subset of the moduli space which, by the results of the forthcoming section, is indeed a connected component of the Gieseker moduli space (cf. Theorem 3.1). The dimension of this component is equal to 6, since if two surfaces $S, S^{\prime}$ are isomorphic, then this isomorphism lifts to a $G$ equivariant isomorphism between $\widehat{S}$ and $\hat{S}^{\prime}$, and we get in particular an isomorphism of the corresponding Albanese surfaces carrying one branch locus $D$ to the other $D^{\prime}$. It is now easy to see that, since we have normalized the line bundle $\mathbb{L}$, the morphism of the base of the rational family to the moduli space is quasi finite.

We shall show that for each canonical model $X$ the base $\mathfrak{B}_{X}$ of the Kuranishi family of deformations of $X$ is smooth of dimension 6 . For this it suffices to show that the dimension of the Zariski tangent space to $\mathfrak{B}_{X}$ is at most 6 , since we already saw that $\operatorname{dim}\left(\mathfrak{B}_{X}\right) \geq 6$.

In fact we could also show that for each canonical model $X$ the above 6-dimensional family induces a morphism $\psi$ of the smooth rational base whose KodairaSpencer map is an isomorphism, whence $\psi$ yields an isomorphism of the base with $\mathfrak{B}_{X}$.

Observe moreover that the assertion about the normality of this component of the Gieseker moduli space follows right away from the fact that the moduli space $\mathfrak{M}_{\chi, K^{2}}$ is locally analytically isomorphic to the quotient of the base of the Kuranishi family by the action of the finite group $\operatorname{Aut}(X)$. Indeed, a quotient of a normal 
space is normal, and the local ring of a complex algebraic variety is normal if its corresponding analytic algebra is normal.

Let now $X=\hat{X} / G$ be the canonical model of a Keum-Naie surface. Note that

$$
\operatorname{Ext}^{1}\left(\Omega_{X}^{1}, \mathcal{O}_{X}\right)=\operatorname{Ext}^{1}\left(\Omega_{\hat{X}}^{1}, \mathcal{O}_{\hat{X}}\right)^{G}
$$

and that

$$
\mathfrak{B}_{X}=\mathfrak{B}_{\hat{X}} \cap \operatorname{Ext}^{1}\left(\Omega_{X}^{1}, \mathcal{O}_{X}\right)=\mathfrak{B}_{\hat{X}} \cap \operatorname{Ext}^{1}\left(\Omega_{\hat{X}}^{1}, \mathcal{O}_{\hat{X}}\right)^{G} .
$$

In order to conclude the proof, it suffices therefore to show that

$$
\mathfrak{B}_{\hat{X}}=\operatorname{Ext}^{1}\left(\Omega_{\hat{X}}^{1}, \mathcal{O}_{\hat{X}}\right),
$$

which shows that $\mathfrak{B}_{\hat{X}}$ is smooth.

We consider then $\hat{X}$ as a double cover of its Albanese variety $A$, and observe that the family of such double covers of a principally polarized abelian surface has dimension equal to $18=3+15$, since abelian surfaces depend on three moduli, and the branch divisor $D$ varies in a linear system of projective dimension $\frac{1}{2} D^{2}-1=16-1=15$ (observe that changing the divisor class to an algebraically equivalent one can be achieved by a translation, which does not change the isomorphism class of the double cover).

Hence we are done once we show that $\operatorname{dim} \operatorname{Ext}_{\mathcal{O}_{\hat{X}}}^{1}\left(\Omega_{\hat{X}}^{1}, \mathcal{O}_{\hat{X}}\right)=18$. This is the content of the following proposition, where we split all the relevant cohomology groups in eigenspaces for the action of the group $\mathbb{Z} / 2 \mathbb{Z}$ generated by the covering involution for the Albanese morphism.

Proposition 2.3. (1) $\operatorname{dim} \operatorname{Ext}_{\mathcal{O}_{\hat{X}}}^{1}\left(\Omega_{\hat{X}}^{1}, \mathcal{O}_{\hat{X}}\right)^{+}=18$;

(2) $\operatorname{dim} \operatorname{Ext}_{\mathcal{O}_{\hat{X}}}^{1}\left(\Omega_{\hat{X}}^{1}, \mathcal{O}_{\hat{X}}\right)^{-}=0$;

(3) $\operatorname{dim} \operatorname{Ext}_{\mathcal{O}_{\hat{X}}}^{2}\left(\Omega_{\hat{X}}^{1}, \mathcal{O}_{\hat{X}}\right)^{+}=2$;

(4) $\operatorname{dim} \operatorname{Ext}_{\mathcal{O}_{\hat{X}}}^{2}\left(\Omega_{\hat{X}}^{1}, \mathcal{O}_{\hat{X}}\right)^{-}=8$.

Proof. Consider the following exact sequence:

$$
\begin{aligned}
0 \rightarrow & \operatorname{Hom}_{\mathcal{O}_{A}}\left(\Omega_{A}^{1}, \mathcal{O}_{A}\right) \rightarrow H^{0}\left(\mathcal{O}_{D}(D)\right) \rightarrow \operatorname{Ext}_{\mathcal{O}_{\hat{X}}^{1}}^{1}\left(\Omega_{\hat{X}}^{1}, \mathcal{O}_{\hat{X}}\right)^{+} \rightarrow \operatorname{Ext}_{\mathcal{O}_{A}}^{1}\left(\Omega_{A}^{1}, \mathcal{O}_{A}\right) \\
& \rightarrow H^{1}\left(\mathcal{O}_{D}(D)\right) \rightarrow \operatorname{Ext}_{\mathcal{O}_{\hat{X}}}^{2}\left(\Omega_{\hat{X}}^{1}, \mathcal{O}_{\hat{X}}\right)^{+} \rightarrow \operatorname{Ext}_{\mathcal{O}_{A}}^{2}\left(\Omega_{A}^{1}, \mathcal{O}_{A}\right) \rightarrow 0
\end{aligned}
$$

for which a convenient reference is [Man01], where the following is proven:

Proposition 2.4. For every locally simple normal flat $(\mathbb{Z} / 2 \mathbb{Z})^{r}$-cover $f: X \rightarrow Y$ there is a $(\mathbb{Z} / 2 \mathbb{Z})^{r}$-equivariant exact sequence of sheaves

$$
0 \rightarrow f^{*} \Omega_{Y}^{1} \rightarrow \Omega_{X}^{1} \rightarrow \bigoplus_{\sigma \in(\mathbb{Z} / 2 \mathbb{Z})^{r}} \mathcal{O}_{R_{\sigma}}\left(-R_{\sigma}\right) \rightarrow 0,
$$


where $R_{\sigma}$ is the divisorial part of $\operatorname{Fix}(\sigma)$.

Moreover, for each $\sigma \in(\mathbb{Z} / 2 \mathbb{Z})^{r}$ and $i \geq 1$, we have

$$
\operatorname{Ext}_{\mathcal{O}_{X}}^{i}\left(\mathcal{O}_{R_{\sigma}}\left(-R_{\sigma}\right), \mathcal{O}_{X}\right) \cong \bigoplus_{\{\chi \mid \chi(\sigma)=0\}} H^{i-1}\left(\mathcal{O}_{D_{\sigma}}\left(D_{\sigma}-\mathscr{L}_{\chi}\right)\right)
$$

Observe that

- $\operatorname{Hom}_{\mathcal{O}_{A}}\left(\Omega_{A}^{1}, \mathcal{O}_{A}\right) \cong \mathbb{C}^{2}$,

- $\operatorname{Ext}_{\mathcal{O}_{A}}^{1}\left(\Omega_{A}^{1}, \mathcal{O}_{A}\right) \cong \mathbb{C}^{4}$,

- $\operatorname{Ext}_{\mathcal{O}_{A}}^{2}\left(\Omega_{A}^{1}, \mathcal{O}_{A}\right) \cong \mathbb{C}^{2}$,

- $H^{1}\left(\mathcal{O}_{D}(D)\right)\left(=H^{1}\left(\mathcal{O}_{D}\left(K_{D}\right)\right)\right) \cong \mathbb{C}$,

- $H^{0}\left(\mathcal{O}_{D}(D)\right)\left(=H^{0}\left(\mathcal{O}_{D}\left(K_{D}\right)\right)\right) \cong \mathbb{C}^{17}$, since $D$ has genus $g=17$ (in fact $\left.2(g-1)=D^{2}=32\right)$.

Note that the map $\lambda: \operatorname{Ext}_{\mathcal{O}_{A}}^{1}\left(\Omega_{A}^{1}, \mathcal{O}_{A}\right) \rightarrow H^{1}\left(\mathcal{O}_{D}(D)\right)$ is the Serre dual of

$$
\mathbb{C} \cong H^{0}\left(D, \mathcal{O}_{D}\right) \rightarrow H^{1}\left(A, \Omega_{A}^{1}\right), \quad 1 \mapsto c_{1}(D),
$$

which is injective. Therefore $\lambda$ is surjective, and part 1) and 2) of the claim follow. that

In order to calculate the antiinvariant parts of $\operatorname{Ext}_{\mathcal{O}_{\hat{X}}}^{i}\left(\Omega_{\hat{X}}^{1}, \mathcal{O}_{\hat{X}}\right), i=1,2$, observe

$$
\operatorname{Ext}_{\mathcal{O}_{\hat{X}}}^{i}\left(\Omega_{\hat{X}}^{1}, \mathcal{O}_{\hat{X}}\right)^{-} \cong \operatorname{Ext}_{\mathcal{O}_{A}}^{i}\left(\Omega_{A}^{1}, \mathcal{O}_{A}(-L)\right) .
$$

But

$$
\begin{aligned}
\operatorname{Ext}_{\mathcal{O}_{A}}^{i}\left(\Omega_{A}^{1}, \mathcal{O}_{A}(-L)\right) & \cong H^{i}\left(A, \Theta_{A}(-L)\right) \\
& \cong H^{i}\left(A, \mathcal{O}_{A}(-L)\right)^{\oplus 2} \cong\left(H^{2-i}\left(A, \mathcal{O}_{A}(L)\right)^{\oplus 2}\right)^{\vee}
\end{aligned}
$$

\section{The fundamental group of Keum-Naie surfaces}

In the previous sections we proved that Keum-Naie surfaces form a normal unirational irreducible component of dimension 6 of the Gieseker moduli space. In this section we shall prove that indeed they form a connected component. More generally, we shall prove the following:

Theorem 3.1. Let $S$ be a smooth complex projective surface which is homotopically equivalent to a Keum-Naie surface. Then $S$ is a Keum-Naie surface.

Let $S$ be a smooth complex projective surface with $\pi_{1}(S)=\Gamma$ ( $\Gamma$ being the fundamental group of a Keum-Naie surface). 
Recall that $\gamma_{i}^{2}=e_{i}$ for $i=1,2$. Therefore $\Gamma=\left\langle\gamma_{1}, e_{1}^{\prime}, \gamma_{2}, e_{2}^{\prime}\right\rangle$ and recall that, as we observed in Section 1, we have the exact sequence

$$
1 \rightarrow \mathbb{Z}^{4} \cong\left\langle e_{1}, e_{1}^{\prime}, e_{2}, e_{2}^{\prime}\right\rangle \rightarrow \Gamma \rightarrow(\mathbb{Z} / 2 \mathbb{Z})^{2} \rightarrow 1,
$$

where $\gamma_{1} \mapsto(1,0), \gamma_{2} \mapsto(0,1)$.

We have set $\Lambda_{i}^{\prime}:=\mathbb{Z} e_{i} \oplus \mathbb{Z} e_{i}^{\prime}$, so that $\pi_{1}\left(E_{1}^{\prime} \times E_{2}^{\prime}\right)=\Lambda_{1}^{\prime} \oplus \Lambda_{2}^{\prime}$.

We define also the two lattices $\Lambda_{i}:=\mathbb{Z} \frac{e_{i}}{2} \oplus \mathbb{Z} e_{i}^{\prime}$.

Remark 3.1. 1) $\Gamma$ acts as a group of affine transformations on the lattice $\Lambda_{1} \oplus \Lambda_{2}$.

2) We have an étale double cover $E_{i}^{\prime}=\mathbb{C} / \Lambda_{i}^{\prime} \rightarrow E_{i}:=\mathbb{C} / \Lambda_{i}$, which is the quotient by a semiperiod of $E_{i}^{\prime}$, namely $e_{i} / 2$.

$\Gamma$ has two subgroups of index two:

$$
\Gamma_{1}:=\left\langle\gamma_{1}, e_{1}^{\prime}, e_{2}, e_{2}^{\prime}\right\rangle, \quad \Gamma_{2}:=\left\langle e_{1}, e_{1}^{\prime}, \gamma_{2}, e_{2}^{\prime}\right\rangle,
$$

corresponding to two étale covers of $S: S_{i} \rightarrow S$, for $i=1,2$.

Lemma 3.2. The Albanese variety of $S_{i}$ is $E_{i}$. In particular, $q\left(S_{1}\right)=q\left(S_{2}\right)=1$.

Proof. Denoting the translation by $e_{i}$ by $t_{e_{i}} \in \mathbb{A}(2, \mathbb{C})$ we see that

$$
\gamma_{1} t_{e_{2}}=t_{e_{2}}^{-1} \gamma_{1}, \quad \gamma_{1} t_{e_{2}^{\prime}}=t_{e_{2}^{\prime}}^{-1} \gamma_{1}, \quad \gamma_{1} t_{e_{1}^{\prime}}=t_{e_{1}^{\prime}} \gamma_{1}
$$

This implies that $t_{e_{2}}^{2}, t_{e_{2}^{\prime}}^{2} \in\left[\Gamma_{1}, \Gamma_{1}\right]$, and we get a surjective homomorphism

$$
\Gamma_{1}^{\prime}:=\Gamma_{1} / 2\left\langle e_{2}, e_{2}^{\prime}\right\rangle \cong \Gamma_{1} / 2 \mathbb{Z}^{2} \rightarrow \Gamma_{1}^{\mathrm{ab}}=\Gamma_{1} /\left[\Gamma_{1}, \Gamma_{1}\right] .
$$

Since $\gamma_{1}$ and $e_{1}^{\prime}$ commute, we have that $\Gamma_{1}^{\prime}$ is commutative, hence

$$
\Gamma_{1}^{\prime} \cong\left\langle\gamma_{1}, e_{1}^{\prime}\right\rangle \oplus(\mathbb{Z} / 2 \mathbb{Z})^{2} \cong \mathbb{Z} \frac{e_{1}}{2} \oplus \mathbb{Z} e_{1}^{\prime} \oplus(\mathbb{Z} / 2 \mathbb{Z})^{2}=\Lambda_{1} \oplus(\mathbb{Z} / 2 \mathbb{Z})^{2} .
$$

Since $\Gamma_{1}^{\prime}$ is abelian $\Gamma_{1}^{\prime}=\Gamma_{1}^{\mathrm{ab}}=H_{1}\left(S_{1}, \mathbb{Z}\right)$. This implies that $\operatorname{Alb}\left(S_{1}\right)=\mathbb{C} / \Lambda_{1}=$ $E_{1}$.

The same calculation shows that $\Gamma_{2}^{\mathrm{ab}}=H_{1}\left(S_{2}, \mathbb{Z}\right)=\Lambda_{2} \oplus(\mathbb{Z} / 2 \mathbb{Z})^{2}$, whence $\operatorname{Alb}\left(S_{2}\right)=\mathbb{C} / \Lambda_{2}=E_{2}$.

For the sake of completeness we prove the following

Lemma 3.3. $H_{1}(S, \mathbb{Z})=\Gamma^{\mathrm{ab}}=\mathbb{Z} / 4 \mathbb{Z} \oplus(\mathbb{Z} / 2 \mathbb{Z})^{3}$.

Proof. We have already seen in the proof of Lemma 3.2 that

$$
\begin{array}{ll}
\gamma_{1} t_{e_{2}}=t_{e_{2}}^{-1} \gamma_{1}, & \gamma_{1} t_{e_{2}^{\prime}}=t_{e_{2}^{\prime}}^{-1} \gamma_{1}, \\
\gamma_{2} t_{e_{1}}=t_{e_{1}}^{-1} \gamma_{2}, & \gamma_{2} t_{e_{1}^{\prime}}=t_{e_{1}^{\prime}}^{-1} \gamma_{2},
\end{array}
$$


and moreover, for $i=1,2$, we have that $\gamma_{i}$ commutes with $e_{i}, e_{i}^{\prime}$.

This shows that we have a surjective homomorphism

$$
\Gamma^{\prime}:=\Gamma /\left\langle 2 e_{1}, 2 e_{1}^{\prime}, 2 e_{2}, 2 e_{2}^{\prime}\right\rangle \cong \Gamma / 2 \mathbb{Z}^{2} \rightarrow \Gamma /[\Gamma, \Gamma] .
$$

Since $\gamma_{2} \gamma_{1}=t_{e_{2}} t_{e_{1}}^{-1} \gamma_{1} \gamma_{2}$, it follows that $e_{2}-e_{1} \in[\Gamma, \Gamma]$, whence we have a surjective homomorphism

$$
\Gamma^{\prime \prime}:=\Gamma^{\prime} /\left\langle e_{1}-e_{2}\right\rangle \rightarrow \Gamma /[\Gamma, \Gamma],
$$

and it is easy to see that the homomorphism $\psi: \Gamma^{\prime \prime} \rightarrow \mathbb{Z} / 4 \mathbb{Z} \oplus(\mathbb{Z} / 2 \mathbb{Z})^{3}$, given by

$$
\begin{aligned}
\psi\left(\bar{\gamma}_{1}\right) & =(1,0,0,0), \quad \psi\left(\bar{\gamma}_{2}\right)=(1,1,0,0), \\
\psi\left(\bar{e}_{1}^{\prime}\right) & =(0,0,1,0), \quad \psi\left({\overline{e^{\prime}}}_{2}\right)=(0,0,0,1) .
\end{aligned}
$$

is well defined and is an isomorphism. This shows the claim.

Let $\widehat{S} \rightarrow S$ be the étale $(\mathbb{Z} / 2 \mathbb{Z})^{2}$-covering which is associated to $\Lambda_{1}^{\prime} \oplus \Lambda_{2}^{\prime}=$ $\left\langle e_{1}, e_{1}^{\prime}, e_{2}, e_{2}^{\prime}\right\rangle \triangleleft \Gamma$. Since $\widehat{S} \rightarrow S_{i} \rightarrow S$, and $S_{i}$ maps to $E_{i}$ (via the Albanese map), we get a morphism

$$
f: \widehat{S} \rightarrow E_{1} \times E_{2}=\mathbb{C} / \Lambda_{1} \times \mathbb{C} / \Lambda_{2}
$$

Then $f$ factors through the Albanese map of $\widehat{S}$ : but, since the fundamental group of $\widehat{S}$ equals $\Lambda_{1}^{\prime} \oplus \Lambda_{2}^{\prime}$, and the covering of $E_{1} \times E_{2}$ associated to $\Lambda_{1}^{\prime} \oplus \Lambda_{2}^{\prime} \leq \Lambda_{1} \oplus \Lambda_{2}$ is $E_{1}^{\prime} \times E_{2}^{\prime}$, we see that $f$ factors through $E_{1}^{\prime} \times E_{2}^{\prime}$ and that the Albanese map of $\widehat{S}$ is $\hat{\alpha}: \widehat{S} \rightarrow E_{1}^{\prime} \times E_{2}^{\prime}$.

We will conclude the proof of Theorem 3.1 with the following

Proposition 3.4. Let $S$ be a smooth complex projective surface, which is homotopically equivalent to a Keum-Naie surface. Let $\widehat{S} \rightarrow S$ be the étale $(\mathbb{Z} / 2 \mathbb{Z})^{2}$-cover associated to $\left\langle e_{1}, e_{1}^{\prime}, e_{2}, e_{2}^{\prime}\right\rangle \triangleleft \Gamma$ and let

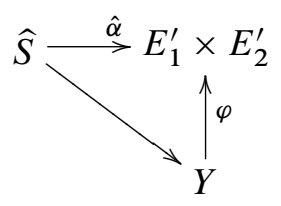

be the Stein factorization of the Albanese map of $\widehat{S}$.

Then $\varphi$ has degree 2 and $Y$ is a canonical model of $\hat{S}$.

Corollary 3.5. $Y$ is a finite double cover of $E_{1}^{\prime} \times E_{2}^{\prime}$ branched on a divisor of type $(4,4)$.

This completes the proof of Theorem 3.1. 
Proof of Proposition 3.4. Consider the Albanese map $\hat{\alpha}: \hat{S} \rightarrow E_{1}^{\prime} \times E_{2}^{\prime}$. Then we calculate the degree of the Albanese map as the index of a certain subgroup of $H^{4}(\widehat{S}, \mathbb{Z})$, namely:

$$
\begin{aligned}
\operatorname{deg}(\hat{\alpha}) & =\left[H^{4}(\hat{S}, \mathbb{Z}): \hat{\alpha}^{*} H^{4}\left(E_{1}^{\prime} \times E_{2}^{\prime}, \mathbb{Z}\right)=\wedge^{4} \hat{\alpha}^{*} H^{1}\left(E_{1}^{\prime} \times E_{2}^{\prime}, \mathbb{Z}\right)\right] \\
& =\left[H^{4}(\hat{S}, \mathbb{Z}): \wedge^{4} H^{1}(\hat{S}, \mathbb{Z})\right] .
\end{aligned}
$$

But since $S$ is homotopically equivalent to a Keum-Naie surface $S^{\prime}$, also $\widehat{S}$ is homotopically equivalent to the étale $(\mathbb{Z} / 2 \mathbb{Z})^{2}$-covering $\hat{S}^{\prime}$ of $S^{\prime}$. Since the $\left[H^{4}(\widehat{S}, \mathbb{Z})\right.$ : $\left.\wedge^{4} H^{1}(\widehat{S}, \mathbb{Z})\right]$ is a homotopy invariant, and the degree of the Albanese map of $\widehat{S}^{\prime}$ is two, it follows that $\operatorname{deg}(\hat{\alpha})=2$.

It remains to show that $Y$ has only rational double points. This follows from the following lemma.

Lemma 3.6. Let $A$ be an abelian surface and let $\widehat{S}$ be a surface with $K_{\hat{S}}^{2}=16$ and $\chi(\widehat{S})=4$. Moreover, let $\varphi: \widehat{S} \rightarrow A$ be a generically finite morphism of degree 2 . Then the branch divisor of $\varphi$ has only non essential singularities (i.e., the local multiplicities of the singular points are $\leq 3$, and for each infinitely near point we have multiplicity at most two, cf. [Hor78]); equivalently, if

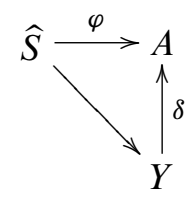

is the Stein factorization, then $Y$ has at most rational double points as singularities.

Proof. We use the notation and results on double covers due to E. Horikawa (cf. [Hor78]). Consider the following diagram:

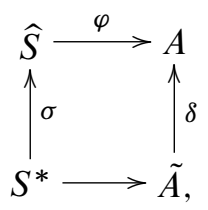

where $S^{*} \rightarrow A$ is the so-called canonical resolution in the terminology of Horikawa.

This means that $\tilde{A} \rightarrow A$ is a minimal sequence of blow ups such that the reduced transform of the branch divisor of $\varphi$ is smooth, so that $S^{*} \rightarrow \tilde{A}$ is a finite double cover with $S^{*}$ smooth, and $S^{*} \rightarrow \widehat{S}$ is a sequence of blow ups of smooth points.

Then we have the following formulae:

$$
\begin{aligned}
K_{S^{*}}^{2} & =K_{\widehat{S}}^{2}-t=2\left(K_{A}+\mathscr{L}\right)^{2}-2 \sum\left(\left[\frac{m_{i}}{2}\right]-1\right)^{2}, \\
\chi\left(S^{*}\right) & =\chi(\widehat{S})=\frac{1}{2} \mathscr{L}\left(K_{A}+\mathscr{L}\right)-\frac{1}{2} \sum\left[\frac{m_{i}}{2}\right]\left(\left[\frac{m_{i}}{2}\right]-1\right),
\end{aligned}
$$


where $t$ is the number of points on $\widehat{S}$ blown up by $\sigma, \mathcal{O}_{A}(2 \mathscr{L}) \cong \mathcal{O}_{A}(B)$, where $B$ is the branch divisor of the (singular) double cover $Y \rightarrow A$. Finally $m_{i} \geq 2$ is the multiplicity of the branch curve in the $i$-th center of the successive blow up of $A$. For details we refer to [Hor78].

Notice that $Y$ has R.D.P.s if and only if $\xi_{i}:=\left[\frac{m_{i}}{2}\right]=1$ for each singular point (and for all infinitely near points).

In our situation, the above two equations read:

$$
\begin{gathered}
K_{S^{*}}^{2}=16-t=2 \mathscr{L}^{2}-2 \sum\left(\xi_{i}-1\right)^{2}, \\
\chi(\widehat{S})=4=\frac{1}{2} \mathscr{L}^{2}-\frac{1}{2} \sum \xi_{i}\left(\xi_{i}-1\right) .
\end{gathered}
$$

This implies that

$$
2 \mathscr{L}^{2}-2 \sum\left(\xi_{i}-1\right)^{2}+t=16=2 \mathscr{L}^{2}-2 \sum \xi_{i}\left(\xi_{i}-1\right),
$$

or, equivalently,

$$
t=-2 \sum\left(\xi_{i}-1\right)
$$

Since $\xi_{i} \geq 1$ this is only possible iff $\xi_{i}=1$ for all $i$ and $t=0$.

Remark 3.2. Note that the above equations also imply that in the case $A=E_{1}^{\prime} \times E_{2}^{\prime}$, $\mathscr{L}$ has to be of type $(2,2)$ or $(1,4)$ (resp. $(4,1))$. But a divisor of type $(1,4)$ cannot be $(\mathbb{Z} / 2 \mathbb{Z})^{2}$ invariant. This proves the above corollary.

In fact, we conjecture the following to hold true:

Conjecture 3.7. Let $S$ be a minimal smooth projective surface such that

i) $K_{S}^{2}=4$,

ii) $\pi_{1}(S) \cong \Gamma$.

Then $S$ is a Keum-Naie surface.

In fact, we can prove

Theorem 3.8. Let $S$ be a minimal smooth projective surface such that

i) $K_{S}^{2}=4$,

ii) $\pi_{1}(S) \cong \Gamma$,

iii) there is a deformation of $S$ having ample canonical bundle.

Then $S$ is a Keum-Naie surface.

Before proving the above theorem, we recall the following results: 
Theorem 3.9 (Severi's conjecture, [Par05]). Let $S$ be a minimal smooth projective surface of maximal Albanese dimension (i.e., the image of the Albanese map is a surface): then $K_{S}^{2} \geq 4 \chi(S)$.

M. Manetti proved Severi's inequality under the stronger assumption that $K_{S}$ is ample, but he also gave a description of the limit case $K_{S}^{2}=4 \chi(S)$, which will be crucial for our result.

Theorem 3.10 (M. Manetti,[Man03]). Let $S$ be a minimal smooth projective surface of maximal Albanese dimension with $K_{S}$ ample: then $K_{S}^{2} \geq 4 \chi(S)$, and equality holds if and only if $q(S)=2$, and the Albanese map $\alpha: S \rightarrow \operatorname{Alb}(S)$ is a finite double cover.

Proof of Theorem 3.8. We know that there is an étale $(\mathbb{Z} / 2 \mathbb{Z})^{2}$-cover $\hat{S}$ of $S$ with Albanese map $\hat{\alpha}: \widehat{S} \rightarrow E_{1}^{\prime} \times E_{2}^{\prime}$. The Albanese map of $\widehat{S}$ must be surjective, otherwise the Albanese image, by the universal property of the Albanese map, would be a curve $C$ of genus 2. But then we would have a surjection $\pi_{1}(\widehat{S}) \rightarrow \pi_{1}(C)$, which is a contradiction since $\pi_{1}(\hat{S})$ is abelian and $\pi_{1}(C)$ is not abelian.

Note that $K_{\widehat{S}}^{2}=4 K_{S}^{2}=16$. By Severi's inequaltiy, it follows that $\chi(\hat{S}) \leq 4$, but since $1 \leq \chi(S)=\frac{1}{4} \chi(\widehat{S})$, we have $\chi(\widehat{S})=4$. Since $S$ deforms to a surface with $K_{S}$ ample, we can apply Manetti's result and obtain that $\hat{\alpha}: \hat{S} \rightarrow E_{1}^{\prime} \times E_{2}^{\prime}$ has degree 2, and we conclude as before.

It seems reasonable to conjecture (cf. [Man03]) the following, which would obviously imply our conjecture 3.7 .

Conjecture 3.11. Let $S$ be a minimal smooth projective surface of maximal Albanese dimension. Then $K_{S}^{2}=4 \chi(S)$ if and only if $q(S)=2$, and the Albanese map has degree 2 .

Remark 3.3. 1) In $[\mathrm{Ke}]$ the author proves that Bloch's conjecture holds, i.e., $A_{0}(S)=$ $\mathbb{Z}$, for the family of surfaces he constructs. Since Keum constructs only a 4-dimensional subfamily of the connected component of the moduli space, this does not imply that Bloch's conjecture holds for all Keum-Naie surfaces. Nevertheless, exactly the same proof holds in the general case, thereby showing that Bloch's conjecture holds true for all Keum-Naie surfaces.

\section{The bicanonical map of Keum-Naie surfaces}

It is shown in [Nai94] that the bicanonical map of a Keum-Naie surface is base point free and has degree 4. Moreover, in [ML-P02], the authors show that the bicanonical image of a Keum-Naie surface is a rational surface, and the bicanonical morphism 
factors through the double cover $S \rightarrow Y$, where $Y=\left(E_{1}^{\prime} \times E_{2}^{\prime}\right) /(\mathbb{Z} / 2 \mathbb{Z})^{2}$ is an 8-nodal Enriques surface. More precisely they show the following (cf. [ML-P02], 5.2.): minimal surfaces $S$ of general type with $p_{g}=0$ and $K^{2}=4$ having an involution $\sigma$ such that

i) $S / \sigma$ is birational to an Enriques surface and

ii) the bicanonical map is composed with $\sigma$

are precisely the Keum-Naie surfaces.

As a corollary of our very explicit description of Keum-Naie surfaces we prove the following

Theorem 4.1. The bicanonical map of a Keum-Naie surface is a finite iterated double covering of the 4-nodal Del Pezzo surface $\Sigma \subset \mathbb{P}^{4}$ of degree 4, the complete intersection of the following two quadric hypersurfaces in $\mathbb{P}^{4}$ :

$$
Q_{1}=\left\{z_{0} z_{3}-z_{1} z_{2}=0\right\}, \quad Q_{2}=\left\{z_{4}^{2}-z_{0} z_{3}=0\right\} .
$$

Proof. Observe first of all that $H^{0}\left(2 K_{S}\right) \cong H^{0}\left(2 K_{\widehat{S}}\right)^{++}$. The standard formulae for the bicanonical system of a double cover allow to decompose $H^{0}\left(2 K_{\widehat{S}}\right)$ as the direct sum of the invariant part $U$ and the anti-invariant part $U^{\prime}$ for the covering involution of the Albanese map.

We have then $H^{0}\left(2 K_{\widehat{S}}\right)=U \oplus U^{\prime}$, where

and

$$
U:=\left\{\Phi\left(z_{1}, z_{2}\right) \frac{\left(d z_{1} \wedge d z_{2}\right)^{\otimes 2}}{w^{2}}\right\}=\hat{\alpha}^{*} H^{0}\left(\mathcal{O}_{E_{1}^{\prime} \times E_{2}^{\prime}}(D)\right)
$$

$$
U^{\prime}:=\left\{\Psi\left(z_{1}, z_{2}\right) \frac{\left(d z_{1} \wedge d z_{2}\right)^{\otimes 2}}{w}\right\}=\hat{\alpha}^{*} H^{0}\left(\mathcal{O}_{E_{1}^{\prime} \times E_{2}^{\prime}}(L)\right) .
$$

Here $\Phi\left(z_{1}, z_{2}\right)=\Phi_{1}\left(z_{1}\right) \Phi_{2}\left(z_{2}\right)$ is a section of $\mathbb{L}^{\otimes 2}=\mathcal{O}_{E_{1}^{\prime} \times E_{2}^{\prime}}(D)$, whereas $\Psi\left(z_{1}, z_{2}\right)=\Psi_{1}\left(z_{1}\right) \Psi_{2}\left(z_{2}\right)$ is a section of $\mathbb{L}=\mathcal{O}_{E_{1}^{\prime} \times E_{2}^{\prime}}(L)$.

Since however $w$ is an eigenvector for $G$ with character of type $(-,+)$, it follows that $w^{2}$ is a $G$-invariant, and, moreover, $U^{++}=\hat{\alpha}^{*} H^{0}\left(\mathcal{O}_{E_{1}^{\prime} \times E_{2}^{\prime}}(D)\right)^{++}$, while $U^{\prime++}=\hat{\alpha}^{*} H^{0}\left(\mathcal{O}_{E_{1}^{\prime} \times E_{2}^{\prime}}(L)\right)^{-+}$.

By the formulae that we developed in Lemma 1.1 the second space is equal to 0 , while the formulae developed in Section 2 show that

$$
U^{++}=\left(V_{1}^{++} \otimes V_{2}^{++}\right) \oplus\left(V_{1}^{--} \otimes V_{2}^{--}\right) \cong \mathbb{C}^{4} \oplus \mathbb{C} .
$$

The first consequence of this calculation is that the composition of $\widehat{S} \rightarrow S$ with the bicanonical map of $S$ factors through the product $E_{1}^{\prime} \times E_{2}^{\prime}$.

Moreover, these sections are invariant for the action of the group $G$, and further for the action of the automorphism

$$
g^{\prime}\left(z_{1}, z_{2}\right):=\left(-z_{1}+\frac{e_{1}}{2}, z_{2}\right)
$$


(observe that $G$ and $g^{\prime}$ are contained in $(\mathbb{Z} / 2 \mathbb{Z})^{2} \oplus(\mathbb{Z} / 2 \mathbb{Z})^{2}$ ).

Whence the above composition factors through the $(\mathbb{Z} / 2 \mathbb{Z})$ quotient $\Sigma$ of the Enriques surface $\left(E_{1}^{\prime} \times E_{2}^{\prime}\right) / G$ by the action of $g^{\prime}$.

$\Sigma$ is a double cover of $\mathbb{P}^{1} \times \mathbb{P}^{1}$ ramified in the union of two vertical plus two horizontal lines. The subspace $\left(V_{1}^{++} \otimes V_{2}^{++}\right)$is the pull back of the hyperplane series of the Segre embedding of $\mathbb{P}^{1} \times \mathbb{P}^{1}$, thus we get a basis of sections $z_{0}, z_{1}, z_{2}$, $z_{3}$ satisfying $z_{0} z_{3}-z_{1} z_{2}=0$.

We can complete these to a basis of $H^{0}\left(2 K_{S}\right)$ by choosing $z_{4}$ such that $z_{4}^{2}=z_{0} z_{3}$.

Since $H^{0}\left(\mathcal{O}_{E_{1}^{\prime} \times E_{2}^{\prime}}(D)\right)^{++}$is base point free, the bicanonical map is a morphism, factoring through the double cover $S \rightarrow Y$ and the double cover $Y \rightarrow \Sigma$.

It is immediate to see that $\left(z_{0}, z_{1}, z_{2}, z_{3}, z_{4}\right)$ yield an embedding of $\Sigma$. We get a complete intersection of degree 4 , hence a Del Pezzo surface of degree 4 . The four nodes, which correspond to the 4 points where the 4 lines of the branch locus meet, are seen to be the 4 points

$$
\begin{aligned}
& z_{4}=z_{1}=z_{2}=z_{3}=0, \\
& z_{4}=z_{1}=z_{2}=z_{0}=0, \\
& z_{4}=z_{0}=z_{3}=z_{1}=0, \\
& z_{4}=z_{0}=z_{3}=z_{2}=0 .
\end{aligned}
$$

\section{References}

[BC10] I. Bauer and F. Catanese, Burniat surfaces II: secondary Burniat surfaces form three connected components of the moduli space. Invent. Math. 180 (2010), 559--588.

[BC11] I. Bauer and F. Catanese, Burniat surfaces I: fundamental groups and moduli of primary Burniat surfaces. In Classification of algebraic varieties. EMS Ser. Congr. Rep., Eur. Math. Soc., Zürich 2011, 49-76.

[BCGP09] I. Bauer, F. Catanese, F. Grunewald, and R. Pignatelli, Quotients of a product of curves by a finite group and their fundamental groups. Amer. J. Math., to appear. arXiv:0809.3420

[Brie68] E. Brieskorn, Die Auflösung der rationalen Singularitäten holomorpher Abbildungen. Math. Ann. 178 (1968), 255-270. Zbl 0159.37703 MR 0233819

[Brie71] E. Brieskorn, Singular elements of semi-simple algebraic groups. In Actes du Congrès International des Mathématiciens (Nice, 1970), Tome 2, Gauthier-Villars, Paris 1971, 279-284. Zbl 0223.22012 MR 0437798

[Cat89] F. Catanese, Everywhere non reduced moduli spaces. Invent. Math. 98 (1989), 293-310. Zbl 0701.14039 MR 1016266

[Cat99] F. Catanese, Singular bidouble covers and the construction of interesting algebraic surfaces. In Algebraic geometry: Hirzebruch 70 (Warsaw, 1998), Contemp. Math. 241, Amer. Math. Soc., Providence, RI, 1999, 97-120. Zbl 0964.14012 MR 1718139 
[Cat08] F. Catanese, Differentiable and deformation type of algebraic surfaces, real and symplectic structures. In Symplectic 4-manifolds and algebraic surfaces, Lecture Notes in Math. 1938, Springer-Verlag, Berlin 2008, 55-167. Zbl 1145.14001 MR 2463711

[Gie77] D. Gieseker, Global moduli for surfaces of general type. Invent. Math. 43 (1977), 233-282. Zbl 0389.14006 MR 0498596

[Hor78] E. Horikawa, Algebraic surfaces of general type with small $c_{1}^{2}$. III. Invent. Math. 47 (1978), 209-248. Zbl 0409.14005 MR 501370

[Ke] Y. H. Keum, Some new surfaces of general type with $p_{g}=0$. Unpublished manuscript, 1988.

[M-M80] R. Mandelbaum and B. Moishezon, On the topology of simply connected algebraic surfaces. Trans. Amer. Math. Soc. 260 (1980), 195-222. Zbl 0465.57014 MR 570786

[Man01] M. Manetti, On the moduli space of diffeomorphic algebraic surfaces. Invent. Math. 143 (2001), 29-76. Zbl 1060.14520 MR 1802792

[Man03] M. Manetti, Surfaces of Albanese general type and the Severi conjecture. Math. Nachr. 261/262 (2003), 105-122. Zbl 1044.14017 MR 2020390

[ML-P02] M. Mendes Lopes and R. Pardini, Enriques surfaces with eight nodes. Math. Z. 241 (2002), 673-683. Zbl 1012.14013 MR 1942235

[Nai94] D. Naie, Surfaces d'Enriques et une construction de surfaces de type général avec $p_{g}=$ 0. Math. Z. 215 (1994), 269-280. Zbl 0791.14016 MR 1259462

[Par05] R. Pardini, The Severi inequality $K^{2} \geq 4 \chi$ for surfaces of maximal Albanese dimension. Invent. Math. 159 (2005), 669-672. Zbl 1082.14041 MR 2125737

[Tju70] G. N. Tjurina, Resolution of singularities of plane deformations of double rational points. Funkstional. Anal. i Prilozhen. 4 (1970), 77-83; English transl. Functional Anal. Appl. 4 (1970), 68-73. Zbl 0221.32008 MR 0267129

Received September 9, 2009; revised February 17, 2010

I. Bauer, F. Catanese, Lehrstuhl Mathematik VIII, Mathematisches Institut der Universität Bayreuth, NW II, Universitätsstr. 30, 95447 Bayreuth, Germany

E-mail: ingrid.bauer@uni-bayreuth.de; fabrizio.catanese@uni-bayreuth.de 